\title{
Relação das obras entradas na Biblioteca da Faculdade durante o periodo compreendido entre 15 de Fevereiro e 15 de Maio de 1934
}

\section{OBRAS GERAIS (0)}

Relatorios, Revistas, Dicionarios, etc.

Anuario Forense-Belo Horizonte, 1934 -1 vol.-Doação.

Anais da Escola de Minas de Ouro Preto, n. 24-Ouro Preto, 1933-1 vol.Permuta.

Anais do Museu Paulista-1933-São Paulo, 1933-1 vol.-Permuta.

Anales de la Universidad de La Republica Oriental del Uruguay-1933Montevideu, 1933-3 vols. -Permuta.

Annals (The)-New York, 1934-1 vol. Compra.

Annuario della Universitá degli studi di Cagliari-1932/33-Cagliari, 1933 -1 vol.--Permuta.

Annuario della Universitá degli studi di Padova-1932/33-Padova, 19331 vol.-Permuta.

Archives de Philosophie du Droit et de Sociologie juridique-Segundo semestre 1933-Paris, 1933-1 vol-Compra.

Archivio di psichiatria, scienze penale ed antropologia criminale-1889 a 1895-15 vols.-Doação.

Arquivos de medicina legal e identificação-vol. 8-Rio de Janeiro, 19341 vol.-Permuta.
Boletin mensual del Seminario de Ciencias juridicas $\mathbf{y}$ sociales-Buenos $\mathrm{Ai}$ res, 1933-1 vol.-Permuta.

Boletim do Instituto da Ordem dos Advogados do Rio Grande do Sul1928 a 1933 -Porto Alegre-6 vols.Permuta.

Boletim do Ministerio da Agricultura -1933-Rio de Janeiro, 1933-1 vol. -Doação

Bulletin of Vassar College-1929/33New York-4 vols.-Doação.

Catalogo da Livraria Coelho (Brasiliana)-Lisboa, 1930-1 vol.-Doação.

Catalogue des livrés imprimés de la Bibliothèque du Roy-Paris,1750—2 vols.-Doação.

Ciencia do Direito-Vol. $1-$ Rio de Janeiro, 1934-1 vol-Compra.

Economiste Français (L') - Segundo semestre 1933-Paris, 1933-1 vol.Compra.

Edgard Fontoura-A Biblioteca Rio Grandense-Rio Grande, 1933-1 vol. -Doação.

Eciclopaedia of the Social Sciencesvol. XII-New York, 1934-1 volCompra.

Gazeta da Relação de Lisboa-Ano 1933 -Lisboa, 1933-1 vol.-Doação.

International Conciliation-Documents for the year-New York, 1933-1 vol. -Doação. 
Juarez Tavora-O ministerio da Agricultura na vigência do Govêrno Proiisorio-Rio de Janeiro, 1933-1 vol. -Doação.

Lista de las ultimas obras publicadas sobre industria ingresadas en la Biblioteca Nacional-Buenos Aires, 1933 -1 vol.--Permuta.

Ministerio (O) do Trabalho no Governo Provisorio-Rio de Janeiro, 1933 -1 vol.-Doação.

Mois (Le) - 1934 -3 vols.-Doação.

Patentes o Marcas-vol. III-São Paulo, 1934-1 vol.- Permuta.

Receuil des Cours de l'Académie de Droit International de la Haye-Tomo IV—Paris, 1924-1 vol.—Doação.

Regimento interno da Escola Livre de Sociologia e Politica de São PauloSão Paulo, 1934-1 folh.-Doação.

Relatorio do Colegio Pedro II-Rio de Janeiro, 1933-1 vol. Doação.

Relatorios apresentados ao Presidente da Republica dos Estados Unidos do Brasil pelo Ministro das Relações Exteriores-1922 a 1929-Rio de Janeiro-14 vols.-Daação.

Relatorio da Chefatura de Policia do Estado de São Paulo-São Paulo, 1934 -1 vol.—Doação.

Relatorios do Ministerio da Fazenda1926 e 1933 - Rio de Janeiro-2 vols. —Doação.

Relatorios do Ministerio da Guerra1918 a 1926 -Rio de Janeiro-6 vols. -Doação.

Relatorios do Ministerio da Marinha1910 a $1930 \longrightarrow$ Rio de Janeiro-24 vols. $\rightarrow$ Doação

Relatorio do Ministerio da Viação1922-Rio de Janeiro, 1924-1 vol.Doação.

Relatorios da Secretaria do Interior do Estado de São Paulo, 1916, 1917, 1920, 1921 a 1923-São (Paulo-6 vols.Doação.

Relatorio da Secretaria e Segurança Publica do Estado de S. Paulo 1927 a 1929-São Paụlo-3 vols.—Doação.

Revista Academica da Faculdade de Direito de Recife-1931-Recife, 19311 vol.-Doação.

Revista de Criminologia, Psiquiatria y
Medicina Legal-Buenos Aires, 19331 vol.-Permuta.

Revista de Direito (Bento de Faria)Rio de Janeiro, 1933-1 vol.-Compra.

Revista juridica da Universidade do Rio de Janeiro-vol. 1-Rio de Janeiro, 1933-1 vol.-Permuta.

Revista do Instituto arqueologico, historico o geografico pernambucanovol. XXXI-Pernambuco, 193.1-1 vol. -Permuta.

Revista de Medicina-Orgão do Centro Academico Oswaldo Cruz-São Paulo, 1932-1 vol.-Doação.

Revista Politecnica-São Paulo, 19331 vol.-Permuta.

Revista do Superior Tribunal de Justiça de Florianopolis-Florianopolis, 1933-1 vol.-Permuta.

Revista dos Tribunais-Vol. 83-São Paulo, 1933—1 vol. -Doação.

Revue d'Economie Politique-Paris, 1933-1 vol.-Compra.

Revue Internacionale de Criminalistique de Lyon-Lyon, 1933-1 vol.Compra.

Revue Trimestrielle de Droit CivilParis, 1933-1 vol.-Compra.

Rivista di Diritto Commerciale-Milano, 1929 a 1931-10 vols.-Compra.

Rivista di Diritto Internazionale-Roma, 1933-1 vol.-Compra.

Scuola Positiva (La)-1892 a 1903-9 vols.-Doação.

Statical Year Book of the League of Nations-Genebra, 1933-1 vol.-Compra.

Vie Intellectuelle (La)-Paris, 1934-2 vols.-Permuta.

Voyages and Travels-Catalogo Maggs Bros. n. 562-Londres 1934-1 vol.Doação.

Year Book of the Carnagie Endowment for International Peace $-1932 / 33$ Washington, 1933-2 vols.—Doação.

\section{FILOSOFIA-PSICOLOGIA-MORAL —etc. (1)}

Almir de Andrade-A verdade contra Freud-Rio de Janeiro, 1933-1 vol. -Compra. 
Baudouin, Charles-Psychologie de la Suggestion et de l'auto-suggestionParis, 1924-1 vol.—Permuta.

Benrubi, J.-Les sources et les courants do la philosophie contemporaine en France $\longrightarrow$ Paris, $1933-2$ vols.-Compra.

Dumas, Georges-Nouveau Traité de Psychologie-Vols. I e II-Paris, 1930, 1932-2 vols.-Compra.

Ellis, Havelock-Précis de Psychologie sexuelle-Paris, 1934-1 vol--Compra,

Franz, Alexander e Staub, Hugo-Psicologia judiciaria-Rio de Janeiro, 1934-1 vol.-Compra.

Freud, S.-O futuro de uma ilusãoRio de Janeiro, $1934-1$ vol.-Permuta.

Freud, S.-Essais de psychanalyse appliquée-Paris, 1933-1 vol.-Permuta.

Goblot, Ed.-El vocabulario filosoficoBarcelona, 1933-1 vol.-Compra.

Giese, Fritz-Psicotecnia-Buenos Aires 1933-1 vol.-Permuta.

Platão-Dialogos-Mexico, 1922-3 vols. Permuta.

Balph, J.-Conhece-te pela psicanalise -São Paulo, 1932-1 vol.-Doação.

Rau Briquet-Preleções de Psicologia Social-São Paulo, 1933-1 vol.-Doação.

Sacker, A.-Du secret professionnel du Banquier-Paris, 1933-1 vol.-Compra.

Velilla, A. S.-O erotismo e a flagelação perante a ciência e a história -São Paulo, 1934-1 vol.-Doação.

Warren, H. C.-Précis de psychologie -Paris, 1923-1 vol.-Permuta.

\section{RELIGIÕES (2)}

Bluteau, Raphael-Sermões panegiricos e doutrinais-Lisboa, 1732-2 vols.Doação.

Brandes, J.-Jesus Cristo é um mito -Rio de Janeiro, 1934-1 vol.-Doaริ̃o.

Fulop-Miller-Les Jésuites et le secret de leur puissance-Paris, 1933-2 vols.-Compra.
Tagore, Rabindranath-A religião do homem-São Paulo, 1934-1 vol.Doação.

CIENCIAS JURIDICAS E SOCIAISSOCIOLOGLA-POLITICA-ESTATISTI. CA-ECONOMICA-DIREITO-MEDICINA LEGAL_EDUCAÇÃO-COSTUMES, etc. (3)

A. Bento de Faria-Aplicação e retroatividade da lei-Rio de Janeiro, 1934 -1 vol.-Compra.

Abilio Pereira de Almeida e José de Queiroz Mattoso-Pratica juridico comercial-São Paulo, 1934-1 vol.Doação.

Albert, Luis J.-Wistoria de la Policia - Buenos Aires, 1913-1 vol.-Doação.

Alexandroff, L.-Traité théorique et pratique des obligations émises par les sociétés-Paris, 1933-1 vol.Compra.

Alfredo Cecilio Lopes-A racionalização dos partidos politicos-São Paulo, 1934-1 vol.-Doação.

Alvarez, Alevandro-Le panamericanisthe $e^{i}$ la sisxième conférence panaméricaine- Paris, 1928-1 vol.-Permuta.

Antonio Paulo da Cunha-Anotaçôes sobre letra de cambio-São Paulo, 1934-1 vol.-Doação.

Arnoldo Medeiros da Fonseca-Teoria geral do direito de retenção-Rio de Janeiro, 1934-1 vol.-Compra.

Artur de Vasconcellos-Irmânia- São Paulo, 1933-1 vol.-Doação.

Bastian, Daniel-La réunion de toutes les actions d'une société entre les mains d'une seule personne-Paris, 1933-1 vol.-Compra.

Berdiaeff, N.-L'homme et la machine - Paris, 1933-1 vol.-Compra.

Bigeard, H.-La règlementation des jeux publics-Angers, 1911-Permuta.

Bodin, Charles-Economie dirigée économie scientifique-Paris, $\quad 1933-1$ vol. -Compra.

Bonn, M. J.-Prosperity-Madrid, 1931 -vol. 1 vol.-Permuta. 
Breard, Lacien-Principes d'économie nationale et internationale-Paris, 1929 -3 vols.-Compra.

Campogrande, V.-Elementi di diritto publico corporativo e di economia politica corporativa-Torino, 19331 vol.-Compra.

Castillo, Carlos Luiz del-Derecho Politico-Madrid, 1934-1 vol.-Doação.

Chesire, G. C.-Il concetto del "trust" secondo la Common Law ingleseTorino, 1933-1 vol.-Doação.

Clovis Bevilaqua-Principios elementares de Direito Internacional Privado, 2. ed.--Rio de Janeiro, 19341 vol.-Doação.

Coleção de leis Municipais Vigentes no Distrito Federal-1893-1931-Rio de Janeiro-4 vols.-Doação.

Comercio (EI) exterior argentino em 1933 y su comparacion con el de 1932 -Buenos Aires, 1934-1 vol.—Doação.

Constitucion politica del Perú-Lima, 1933-1 vol.—Doação.

Davis, Horace, B.-N. R. A.-Fascismo e Comunismo-São Paulo, 1933-1 vol.-Doação.

Didimo Agapito da Veiga-Pareceres do Consultor da Fazenda PublicaRio de Janeiro, 1925-1927-2 vols.Compra.

Diogenes Ribeiro do Valle-Dos processos policiais-São Paulo, 19281 vol.-Doação.

Eduardo de Campos Maia-JulgadosSão Paulo, 1920-1 vol_-Doação.

Einzig, Paul-La crisis economica mundial-Madrid, 1933-1 vol.-Compra.

Einzig Paul-Fundamentos economicos del fascismo-Madrid, 1933- 1 vol. -Compra.

Entidades patrimonais na organização corporativa-Lisboa, 1934-1 vol.Doação.

Escola de Engenharia de Porto Alegre -Porto Alegre, 1928-1 folh.-Doação.

Estatuto do trabalho nacional-Lisboa, 1933-1 folh.-Doação.

F. T. Souza Reis- A depressão economica $\theta$ o Funding Loan-Rio de Janeiro, 1934 -1 vol. DDoação.

Fernando de Magalhães—Obstetricia
forense-Rio de Janeiro, 1933-1 vol.

- Compra.

Finanças dos Estados do Brasil - Rio de Janeiro, 1934-1 vol.-Doação.

Fischer, Irving-L'illusion de la monnaie stable-Paris, 1929-1 vol.Compra.

Garay, Narciso- Año y medio en Genebra-Panamá, 1934-1 vol.-Doaçāo.

Gendini, Sabino A.-Los contratos publicos-Madrid, 1934-1 vol.-Doação.

Gentili, Alberico—De juri belli libri tres-Londres, 1933-2 vols.-Doação.

Ghiron, Mario-Sull'ordinamento della facoltá giuridica-Roma, 1931-1 vol. -Permuta.

Hahnemann Guimarães-Revogação dos atos praticados em fraude dos credores segundo o direito romanoRio de Janeiro, 1930 - 1 vol.-Doação.

Hahnemann Guimarães-Estudos sobre a gestão de negócios-Rio de Janeiro, 1932-1 vol.DDoação.

Henrique Tanner de Abreu-Manual de necropsia forense-Rio de Janeiro, 1930-1 vol.-Compra.

Hilbert - Les vues et les jours de la servitude--Paris, 1933-1 vol-_Compra.

Inquerito do Instituto de Café do Estado de São Paulo-São Paulo, 1933 -7 vols.-Doação.

International Legislation-vols. 1 a 4 Washington, 1931-4 vols.-Doação.

J. do Amaral Gurgel-Do inventario amigavel—São Paulo, 1932-1 vol.Doação.

J. Goncalves Maia-Do fideicommissoRio de Janeiro, 1932-1 vol.-Doação.

J. Gonçalves Maia-Teoria e pratica das procurações-São Paulo, 1927-1 vol. 一Doação.

J. M. de Carvalho Santos-Codigo civil brasileiro interpretado, vols. 2 e 3 Rio de Janeiro, 1934_2 vols.-Compra.

Julgados do Tribunal Superior de Justiça de Alagoas-1919 a 1921-Maceió, 1934-1 vol.-Doação.

Julio de Revoredo-Do delito culposo -São Paulo, 1934, 1 vol.-Doação.

Jurisprudencia Uruguaia-Tomos 8 e $\mathbf{9}$ -Montevideo, 1933-2 vols.-Doação. 
Kelsen-Teoria general del EstadoBarcelona, 1934-1 vol.-Compra.

Labriola, A.-Au dela du capitalisme et du socialisme-Paris, 1932-1 vol. -Compra.

Lasky, Harold-Derecho y politicaMadrid, 1933 1 vol.-Compra.

Lavergne, B.-Le gouvernement des démocraties modernes-Paris, 19331 vol. Compra.

Lee, R. W.-Lezioni sul Impero Britannico-Torino, 1934-1 vol.-Doação.

Legislação Social Trabalhista-Rio de Janeiro, 1934-1. a suplemento-1 vol. Doação.

Leroy-Beaulieu-Les impôts et les revenus en France, en Angleterre et en Allemagne-Paris, 1914—1 vol.-Doaç̃̃o.

Mario de Assis Moura-O reajustamento economico-São Paulo, 1934-1 vol. -Compra.

Martin, Etienne-Précis de médecine légale-lParis, 1932-1 vol.-Compra.

Mirkine - Guétzevitch-Droit constitutionnel international-Paris, 1933-1 vol.-Compra.

Morando, A.-El contrato de cuenta corrente-Barcelona, 1933-1 vol.-Compra.

Natekvicius, L. - Aspect politique et juridique du différend polono-lithuanien-Paris, 1930-1 vol.-Doação.

Niboyet et Gaulé-Receuil de textes usuels de Droit International-Paris, 1929-3 vols.-Permuta.

Noção (A) de resistencia das associações de classe-Lisboa, 1933-1 folh. -Doação.

Molinier, H.-De l'amende fiscaleToulouse, 1909-1 vol.-Permuta.

Oppenheim, L.-International LawNew York, 1920-2 vols.-Permuta.

Ordem (A) corporativa-Lisboa, 19341 folh.Doação.

Organização Corporativa-Lisboa, 1934 - 1 vol.-Doação.

Orue, José Ramon de-Manual de Decho Internacional publico-Madrid, 1934-1 vol. Doação.

Oswaldo Aranha-Discurso sôbre a situação financeira do Brasil, pronunciado na Assembléia Constituinte em
16-3-934-Rio, 1934-1 vol.-Doação.

Palomeque, Rafael Alberto-Desenvolvimiento $y$ estado de la educación primaria em 1932-La Plata, 19331 vol.

Pareceres do Consultor da RepublicaVols. 1 a 11-Rio de Janeiro, 1911 a 1927-11 vols.-Compra.

Paulo Alberto-Juramento-Baía, 1933 -1 vol.-Doação.

Paulo Duarte-Um conluio imoral-São Paulo, 1934-1 vol_-Doação.

Paulo Prado-Paulistica-2." ediçãoRio de Janeiro, 1934-1 vol.-Doação.

Pedro Antonio de Oliveira Ribeiro Neto-OS crimes dos epiléticos-São Paulo, 1934-1 vol.-Doação.

Pedro Teotonio Pereira-As idéias do Estado novo-Lisboa, 1933-1 vol.Doação.

Pinero, Norberto-La letra de cambio ante el derecho internacional privado-Buenos Aires, 1932-1 vol.Compra.

Pirotti, Simon-La clause or devant la loi et les tribunaux-Paris, 1933-1 vol.-Compra.

Planiol, Marcel et Ripert, GeorgesTraite pratique de Droit Civil Francais-vol. XIV-Paris, 1934-1 vol.Compra

Problemas da organização corporativa -Lisboa, 1934-1 folh.-Doação.

Radbruch, Gustavo-Filosofia del Derecho-Madrid, 1933-1 vol--Permuta.

Rafael Correa de Sampaio e Noé Azevedo-A questão da independencia entre as responsabilidades civil e criminal-São Paulo, 1934-1 folh.Doação.

Reca, Graciano-Inamovibilidad de los jueces-Buenos Aires, 1933-1 vol.Doação.

Reginaldo Nunes-Apontamentos sobre a Introdução ao estudo do DireitoSão Paulo, 1934-1 vol.-Compra.

Reid, Helen, D.-International servitude in Law and Practice-Chicago, 19331 vol.-Doação.

Republica Argentina comme paese d'immigrazione-Buenos Aires, 1904-1 vol.-Doação.

Roubier, Paul-Les conflits de lois 
dans le temps-Paris, 1929-1 vol.Compra.

Sagen, Lyder-Dinamarca, pais agricola-São Paulo, 1933-1 vol.-Doação.

Saldana, Quintilliano-Nova criminologia-São Paulo, 1934-1 vol.-Compra.

Stainof, P.-Le fonctionnaire-Paris, 1933-1 vol.-Permuta.

Toulemon, André-Le suffrage familial ou suffrage universel integralParis, 1933, 1 vol.-Compra.

Toulouse (Dr.) - La vie nouvelle-Paris, $s / d-1$ vol.-Permuta.

Tumeneff, I.-Historia do trabalhoSão Paulo, 1934-1 vol.-Doação.

Ulloa, Alberto--El fallo arbitral del presidente de Estados Unidos en la question de Tacna y Arica-Lima, 19251 vol.-Doação.

Vicente Piragibe-Codigos penais estrangeiros-Argentina, Perú, ItaliaRio de Janeiro, 1934-1 vol--Compra.

Weber, Anatole-Traité des Sociètés de capitalisation-Paris, 1931-1 vol.Compra.

Whitton, John-La doctrine de Monroe -Paris, 1933-1 vol.-Compra.

Zubieta, José Antonio-Censo demografico 1930—Panamá, 1931-2 vols. Doação.

\section{FILOLOGIA E LINGUISTICA (4)}

Staaff, Erik-Le Laudario de PiseÉtudo linguistique-Leipzig, 1931-1 vol. - Doação.

Zizenando Camargo-Inventário lexicografico semântico, sintético e estilistico do primeiro livro dos "Annales" de Tacito-S. Paulo, 1934-1 vol. -Doação.

Tabachovitz, A.-Etude sur la langue de la version française des sements de Strabourg-Uppsala, 1932-1 vol.-Permuta.

\section{ctenclas pUras (5)}

Alfredo Ellis Jr.-Populações paulistas-Conferencias mimiografadas-
-S. Paulo, 1933-1 vol.-Doação.

Freud, S.-Totem e tabú-Rio, 19341 vol.-Permuta.

Haøckel, E.-Religião e evolução-Porto, 1919-1 vol.-Permuta.

Haeckel, E.-O monismo-Porto, 19191 vol.-Permuta.

Mendes Correa - Homo - Coimbra, 1926 -1 vol.-Permuta.

Morgan, J. de - L'humanité prehistorique-Paris, 1924—1 vol.-Permuta.

Pierantoni, U.-Compendio de Biologia -Barcelona, 1931-1 vol.-Compra.

Pittard, E.-Les races et l'HistoireParis, 1924-1 vol.-Permuta.

Renato Kehl-Conduta—Rio de Janeiro, 1934-1 vol.—Doação.

\section{Ciencias APlicadas (6)}

A. Almeida Jr.-Fisiologia do Trabalho -Preleções mimiografadas-S. Paulo, 1933-1 vol.-Doação.

Afranio Peixoto-Higiene-Rio, 19302 vols.-Compra.

Eugenio George-Sôros e vacinas-Aspectos da civilização de alienadosRio, 1934-1 vol,-Doação.

Franco, Enrico Emilio-Manuale Atlante di Tecnica delle Autopsie-Milano, 1926-1 vol.-Compra.

Gaspar Ricardo Jr.-Relações entre os transportes ferroviarios e rodoviarios - S. Paulo, 1934-1 vol.-Doação.

João de Aguiar Pupo-A Santa Casa da Misericordia de São Paulo e sua cooperação na obra da profilaxia da lepra-São Paulo, 1934-1 vol.Doação.

Mario Cabral Jr.-A Estrada de Fierro Sorocabana e o seu serviço de coordenação de transportes-S. Paulo, 1934-1 vol.—Doação.

Mass, Lucien - Les névroses et l'Histoire-Paris, 1908-1 vol.-Permuta.

Nelson Ottoni de Rezende-Departamento autonomo de Estradas de rodagem-S. Paulo, 1934-1 vol.-Doação.

R. de Almeida Cunha-Lições de microbiologia-Belo Horizonte, 19321 vol.-Permuta. 
BELAS ARTES (7)

Mario de Andrade-Compendio de Historia da Musica-S. Paulo, 1933-1 vol.-Doação.

\section{LITERATURA}

Afonso de Carvalho-Anchieta escritor e 'poeta-S. Paulo, 1934-1 vol.Doação.

Aristophane - Plutus - Paris, 1897 - 1 vol.-Permuta.

Aristote-Poetique-Paris, 1923-1 vol. -Permuta.

Castro Alves-Obras completas-Rio, 1921-2 vols.-Permuta.

Cicéron-Plaidoyer pour Milon-Paris, 1921-1 vol.-Permuta.

Cicéron-Dialogue sur l'amitié-Paris, 1920-1 vol.--Permuta.

Demosthènes-Discours sur la couronne -Paris, 1916—1 vol. -Permuta.

Duisberg, Karl-Vortrage und reden aus den Jahren-1882-1821-Leipzig, 1923 - 1 vol.-Doação.

Esopo-Fables-Paris, 1920-1 vol.Permuta.

Esprit (L') de Paris--Paris, s/d.--1 vol. Doação.

Esquilo - Tragedias - Mexico, 1923-1 vol.-Permuta.

Euripedes-Aloeste-Paris, s/d.-1 vol. -Permuta.

Euripedes-Electre-Paris, 1893-1 vol. -Permuta.

Euripedes-Tragedias - Mexico, 1921 1 vol.-Permuta.

Genung, J. F. - The working principles of rethoric $-N$. York, 1900-1 vol.-Permuta.

Goomilewsky, Léo-O amor em liberdade -S. Paulo, 1934-1 vol.-Doação.

Horace-Art poétique-Paris, s/d.-1 vol.-Penmuta.

Hugo, Victor-O noventa e tres-S. Paulo, 1932-1 vol.-Doação.

Humberto de Campos-Da seảra de Booz-Rio, 1933-1 vol.-Doação.

Ibsen, Enrique - La comedia del amor -Valencia, s/d-1 vol.-Permuta.

In memoria-Felippe de Oliveira-S. Paulo, 1933-1 vol.-Doação.
Keyserling, H.-Diario de viaje de un filosofo-Madrid, 1928-2 vols.-Permuta.

Lalouel, H. - Le orateurs de La Grande Bretagne depuis le regne de Charles 1er.-Paris, 1841-1 vol.-Doação.

Lucio José dos Santos-Goethe-B. Horizonte, 1933-1 vol.-Permuta.

Meirs, G. e S. M. Darros-O cadaver assassino-S. Paulo, 1932-1 volDoação.

Moreno, J. C.-Frente al mundo-B. Aires, $1933-1$ vol.-Doação.

Pedro Ribeiro de Araujo BittencourtDiscurso-Baia, 1934-1 vol.-Doação.

Phedre—Fables esopique-Paris, 19201 vol.-Permuta.

Picarolo, A.-Entre a ciencia e a arte-S. Paulo, 1934-1 vol.-Doação.

Platão-Fédon-Porto, 1920-1 vol.Permuta.

Platon-Apologie de Socrate-Paris, s/d. -1 vol.-Piermuta.

Platon-Creton-Paris, s/d.--1 vol.Permuta.

Renan, Ernest-Histoire littéraire de la France au 14ème. siècle-Paris, 1865-2 vols.-Permuta.

Ribeiro Couto-Clube das esposas enganadas-Rio, 1933-1 vol.-Permuta.

Segredo Conjugal-Diversos autoresRio, 1932-1 vol.-Doação.

Tolstoi, Leão-Os Cosacos-S. Paulo, 1932—1 vol.—Doação.

Veriaine, Paul-Oeuvres complétesParis, 1926-5 vols.-Permuta.

Verne, Maurice-Mussés de voluptésParis, 1930-1 vol.-Doação.

Wallace, Edgar-O rei da noite-S. Paulo, 1932-1 vol.-Droação.

Xisto-As mil e uma anedotas-S. Paulo, 1926-1 vol.-Doação.

\section{HISTORIA GEOGRAFIA POLTICA - VIAGENS BIOGRAFIA HERALDI- CA, etc. (9)}

Affonso E. Taunay-Historia seiscentista da vila de S. Paulo-Vols. II e IV-São Paulo, 1927-1929-2 vols.Doação.

Agassiz, Lonis--Scientifics results of a journey in Brazil-Boston, 1870-1 vol.-Permuta. 
Aroldo de Azevedo-Geografia Humana-S. Paulo, 1934-1 vol.-Doação.

Cesar Feliciano Xavier-O voadorBartolomeu de Gusmão-Rio, 19311 vol.-Doação.

Cornelio Nepos-Vie des grands capitanies-Paris, 1921-1 vol.-Permuta.

Diplomatic correspondence of the United States-Vols. I e II-Washington, 1932_2 vols.--Doação.

Escuelo (El)-Nacional-B. Aires, 1933 - 1 vol.-Permuta.

Gilberto Freyre-Casa Grande e Senzala-Rio, 1934-1 vol.-Compra.

Homenagem a José Bonifacio no $88^{\circ}$ aniversario da Independencia do Brasil-Rio, 1910—1 vol.-Doação.

Honorio de Sylos-Itararé! Itararé !S. Paulo, 1933-1 vol. -Doação.

J. P. Oliveira Martins-Taboas de cronologia e geografia historica-Lisboa, 1884-1 vol.-Permuta.

João Batista de Sousa Fillho-Aponta- mentos genealogicos-S. Paulo, 1930 -1 vol. -Doação.

Manoel Miranda-O programa de José Bonifacio-Rio, 1911-1 vol.-Doação. Manoel Osorio-A guerra de S. Paulo -S. Paulo, 1932-1 vol.-Permuta.

Orlando Machado-Arquipelago de Fernando de Noronha-1 vol.-Doação.

Paulo Prado-Paulistica-2." ed.-Rio, 1934-1 vol.-Doação.

Palomeque, Rafael Alberto-Sarmiento, otros discursos-La Plata, 1933-1 vol.-Permuta.

Rolland, Romain-Vidas ejemplaresMexico, 1923-1 vol--Permuta.

Vaillant-Coururier-No misterioso pais de Tamerlão-S. Paulo, 1934-1 vol. -Doação.

Xénophon-Extraits memorables--Paris, 1914-1 vol.-Permuta.

Zweig, Stephan-Momentos decisivos da humanidade-Rio, 1934-1 vol.Permuta. 